\title{
Testosterone: Relationships with Metabolic Disorders in Men-An Observational Study from SPECT-China
}

\author{
Jing Cheng, ${ }^{1}$ Bing Han, ${ }^{1}$ Qin Li, ${ }^{1}$ Fangzhen Xia, ${ }^{1}$ Hualing Zhai, ${ }^{1}$ Ningjian Wang, \\ Michael Jensen, ${ }^{2}$ and Yingli $\mathrm{Lu}^{1}$ \\ ${ }^{1}$ Institute and Department of Endocrinology and Metabolism, Shanghai Ninth People's Hospital, \\ Shanghai Jiao Tong University School of Medicine, Shanghai, China \\ ${ }^{2}$ Endocrine Research Unit, Mayo Clinic, Rochester, MN, USA
}

Correspondence should be addressed to Ningjian Wang; wnj486@126.com, Michael Jensen; jensen@mayo.edu, and Yingli Lu; luyingli2008@126.com

Received 17 August 2017; Revised 18 October 2017; Accepted 2 November 2017; Published 3 December 2017

Academic Editor: Mario Maggi

Copyright (C) 2017 Jing Cheng et al. This is an open access article distributed under the Creative Commons Attribution License, which permits unrestricted use, distribution, and reproduction in any medium, provided the original work is properly cited.

\begin{abstract}
Background. The strength of associations between total testosterone (TT) and metabolic parameters may vary in different nature of population structure; however, no study has ever given this information in Chinese population, especially those without metabolic syndrome (MS). We aimed to analyze the association magnitudes between TT and multiple metabolic parameters in general Chinese men. Methods. 4309 men were recruited from SPECT-China study in 2014-2015, which was performed in 22 sites in East China. TT, weight status, and various metabolic parameters were measured. Linear and logistic regressions were used to analyze the associations. Results. Men in lower TT quartiles had worse metabolic parameters including body mass index, triglycerides, HbAlc, and HOMA-IR (all $P$ for trend $<0.001$ ). Body mass index (B $-0.32,95 \% \mathrm{CI}-0.35$ to -0.29 ) and obesity (OR $0.40,95 \% \mathrm{CI} 0.35-0.45$ ) had the largest association magnitude per one SD increment in TT, while blood pressure and hypertension (OR 0.90, 95\%CI 0.84-0.98) had the smallest. These associations also persisted in individuals without metabolic syndrome. Conclusions. Obesity indices had closer relationships with TT than most other metabolic measures with blood pressure the least close. These associations remained robust after adjustment for adiposity and in subjects without metabolic syndrome.
\end{abstract}

\section{Background}

Metabolic disorders, including diabetes, obesity, and fatty liver, have increased dramatically throughout the world [1]. For example, in 2000-2001, the prevalence of metabolic syndrome (MS), as defined by the US National Cholesterol Education Program Adult Treatment Panel III criteria, was $9.8 \%$ in men and $17.8 \%$ in women in Chinese adults aged 30 to 70 years [2]. One decade later, the prevalence had increased to $31.0 \%$ in men and $36.8 \%$ in women [1]. Given the health and economic burden imposed by the soaring prevalence of metabolic disorders, attention should be paid to their factors associated with and in some cases driving and influencing their pathophysiology.
Testosterone, the primary endogenous sex hormone in men, is secreted mainly by the testes and to a small extent by the adrenal glands [3]. In addition to maintaining development of male reproductive tissues and secondary sexual characteristics [4], there is evidence that testosterone is essential for men's health and well-being; insufficient testosterone is associated with metabolic abnormalities, cardiovascular diseases, and so forth [4, 5]. Furthermore, cross-sectional and prospective studies have found that low total testosterone (TT) concentrations are associated with metabolic disorders in men [4-9].

Low TT is common in Chinese men-our previous study found a prevalence of $17.5 \%$ [9]. It is not fully clear whether TT was associated with multiple metabolic disorders and 
parameters in Chinese. Also, the strength of associations may vary across different metabolic parameters in different populations $[5,8]$ and it is important to know which metabolic parameters are more closely related to TT. No data regarding this information is available for a Chinese population. Hence, using data from the Survey on Prevalence in East China for Metabolic Diseases and Risk Factors (SPECT-China), we analyzed the association magnitude between TT and multiple metabolic parameters in general Chinese men.

\section{Methods}

2.1. Participants. The data are from the participants of SPECT-China, a cross-sectional survey in East China (ChiCTR-ECS-14005052). Recruitment and enrollment have been previously described in detail [10-12]. Briefly, it is an ongoing study of prevalence and incidence of metabolic disease including diabetes, obesity, and fatty liver and their potential risk factors in East China, which is also its primary aim. A stratified cluster sampling method was used to select a sample in the general population, which was stratified according to rural/urban area and economic development status in Shanghai, Jiangxi Province, Zhejiang Province, Jiangsu Province, and Anhui Province. Chinese citizens $\geq 18$ years old who had lived in their current area for $\geq 6$ months were selected. We excluded subjects with severe communication problems, acute illness, or who were unwilling to participate. From 2014 January to 2015 December, 10,441 subjects who were 18-93 years old were recruited in the SPECTChina study from 22 sites in Shanghai, Zhejiang Province, Jiangsu Province, Anhui Province, and Jiangxi Province. There were 4309 men. After excluding 9 subjects without TT, data from 4300 men was included in this study.

The study protocol was approved by the Ethics Committee of Shanghai Ninth People's Hospital, Shanghai Jiao Tong University School of Medicine (approval number 2013). All procedures followed were in accordance with the ethical standards of the responsible committee on human experimentation (institutional and national) and with the Helsinki Declaration of 1975, as revised in 2008. Informed consent was obtained from all patients included in the study.

2.2. Measurements. Interview and collection of biological specimens at each site were undertaken with a single assessment protocol. Blood samples were obtained between 7:00 am and 10:00 am after fasting for at least $8 \mathrm{~h}$. Blood was refrigerated immediately after phlebotomy, and after $2-4 \mathrm{~h}$, it was centrifuged and the serum was aliquoted and frozen in a central laboratory. Total $\mathrm{T}$ (Siemens, immulite 2000, Erlangen, Germany) was detected using a chemiluminescence assay. Glycated hemoglobin (HbA1c) was measured by high-performance liquid chromatography (MQ-2000PT, Medconn, Shanghai, China). Fasting plasma glucose (FPG), triglycerides (TG), total cholesterol (TC), high-density lipoprotein (HDL), and low-density lipoprotein (LDL) were measured by a Beckman Coulter AU680 (Brea, USA). Samples with values below the minimal detectable limit were given a value midway between zero and the minimal detectable limit for the analyses [13]. The interassay and intra- assay coefficients of variation were $6.6 \%$ and $5.7 \%$ for total $\mathrm{T}$, respectively. The homeostasis model assessment of insulin resistance (HOMA-IR) was calculated as fasting serum insulin $(\mathrm{mIU} / \mathrm{L}) \times$ FPG $(\mathrm{mmol} / \mathrm{L}) / 22.5$.

Weight (kilograms) and height (centimeters) were measured using a stadiometer and a vertical ruler when subjects wore light clothing without shoes. Body mass index (BMI) was calculated as weight in kilograms divided by height in meters squared. Waist circumference was measured at a level midway between the lowest rib and the iliac crest. Blood pressure was measured using standard methods as described previously [14]. Neck circumference was measured with head erect and eyes facing forward, horizontally at the upper margin of the laryngeal prominence with a flexible tape [15]. Current smoking was defined as having smoked at least 100 cigarettes in one's lifetime and currently smoking cigarettes [14].

2.3. Definition of Variables. Central obesity was defined as a waist circumference $\geq 90 \mathrm{~cm}$ in males [14]. Overweight and obesity were defined based upon BMI measures of $25-29.9 \mathrm{~kg} / \mathrm{m}^{2}$ and $\geq 30 \mathrm{~kg} / \mathrm{m}^{2}$, respectively [14]. Diabetes was determined using a previous diagnosis by health care professionals, FPG level $\geq 7.0 \mathrm{mmol} / \mathrm{L}$, or $\mathrm{HbA} 1_{c} \geq 6.5 \%$. Hypertension was identified by a systolic blood pressure more $\geq 140 \mathrm{mmHg}$, a diastolic blood pressure $\geq 90 \mathrm{mmHg}$, or a self-reported previous diagnosis of hypertension by a physician. According to the modified National Cholesterol Education Program-Adult Treatment Panel III, dyslipidemia was defined as total cholesterol $\geq 6.22 \mathrm{mmol} / \mathrm{L}$, triglycerides $\geq 2.26 \mathrm{mmol} / \mathrm{L}, \mathrm{LDL}-\mathrm{C} \geq 4.14 \mathrm{mmol} / \mathrm{L}$ or $\mathrm{HDL}-$ $\mathrm{C}<1.04 \mathrm{mmol} / \mathrm{L}$, or a self-reported previous diagnosis of hyperlipidemia by physicians [16].

MS was determined based on the International Diabetes Federation criteria (2005) [17]. A male with MS must have abdominal obesity (waist circumference $\geq 90 \mathrm{~cm}$ or BMI $\geq 30 \mathrm{~kg} / \mathrm{m}^{2}$ ) plus any two of the following four parameters: (i) raised $\mathrm{TG} \geq 1.7 \mathrm{mmol} / \mathrm{L}$ or treatment for this dyslipidemia; (ii) reduced HDL-C $<1.03 \mathrm{mmol} / \mathrm{L}$ or treatment for this dyslipidemia; (iii) raised BP: systolic $\mathrm{BP} \geq 130$ or diastolic $\mathrm{BP} \geq 85 \mathrm{mmHg}$ or treatment of hypertension; and (iv) raised $\mathrm{FPG} \geq 5.6 \mathrm{mmol} / \mathrm{L}$ or a history of type 2 diabetes.

2.4. Statistical Analysis. Data analyses were performed using IBM SPSS Statistics, Version 22 (IBM Corporation, Armonk, NY, USA). All analyses were two-sided. A $P$ value $<0.05$ indicated significance. Continuous variables were expressed as the mean \pm standard deviation (SD) and categorical variables as a percentage (\%), respectively. $P$ for trend was calculated by ANOVA and Chi-square test.

TT in our population did not comply with the normal distribution. Prior to regression analyses, TT and metabolic measures (continuous variables) were ln-transformed and scaled to standard deviations (SD). Associations of TT with metabolic measures were analyzed using linear regression models with each metabolic measure as outcome and TT as the explanatory variable. The regression models were adjusted for age, smoking, economic status, and BMI (not for waist and neck circumference and waist-hip ratio). To 
TABLE 1: Characteristics of the participants by quartiles of total testosterone.

\begin{tabular}{|c|c|c|c|c|c|}
\hline & & Total test & $\mathrm{nmol} / \mathrm{L}$ & & \\
\hline & Q1 & Q2 & Q3 & Q4 & $P$ for trend \\
\hline & $\leq 12.60$ & $12.61-15.70$ & $15.71-19.91$ & $\geq 19.92$ & $r$ lor tiend \\
\hline$N$ & 1094 & 1059 & 1072 & 1075 & \\
\hline Age, yr & $53(13)$ & $52(13)$ & $53(14)$ & $58(13)$ & $<0.001$ \\
\hline Body mass index, $\mathrm{kg} / \mathrm{m}^{2}$ & $26.3(3.4)$ & $25.2(3.1)$ & $24.5(3.1)$ & $23.3(3.1)$ & $<0.001$ \\
\hline Waist circumference, $\mathrm{cm}$ & $87.8(9.6)$ & $85.1(9.1)$ & $83.4(9.4)$ & $81.0(9.2)$ & $<0.001$ \\
\hline Neck circumference, $\mathrm{cm}$ & $37.0(3.6)$ & $36.3(3.4)$ & $35.6(3.2)$ & $34.8(2.6)$ & $<0.001$ \\
\hline Waist-hip ratio & $0.91(0.07)$ & $0.89(0.07)$ & $0.89(0.07)$ & $0.88(0.08)$ & $<0.001$ \\
\hline Triglycerides, mmol/L & $2.66(2.92)$ & $1.95(1.74)$ & $1.62(1.19)$ & $1.28(0.70)$ & $<0.001$ \\
\hline $\mathrm{HDL}, \mathrm{mmol} / \mathrm{L}$ & $1.27(0.31)$ & $1.31(0.30)$ & $1.38(0.32)$ & $1.47(0.32)$ & $<0.001$ \\
\hline $\mathrm{LDL}, \mathrm{mmol} / \mathrm{L}$ & $3.11(0.76)$ & $3.06(0.73)$ & $3.10(0.76)$ & $3.06(0.78)$ & 0.40 \\
\hline Total cholesterol, mmol/L & $5.26(1.22)$ & $5.12(1.17)$ & $5.14(1.08)$ & $5.09(0.98)$ & $<0.01$ \\
\hline Fasting plasma glucose, $\mathrm{mmol} / \mathrm{L}$ & $6.09(1.88)$ & $5.72(1.45)$ & $5.56(1.44)$ & $5.45(1.10)$ & $<0.001$ \\
\hline $\mathrm{HbAlc}, \mathrm{mmol} / \mathrm{L}$ & $5.8(1.3)$ & $5.6(1.0)$ & $5.6(1.0)$ & $5.5(0.7)$ & $<0.001$ \\
\hline HOMA-IR & $2.11(3.24)$ & $1.56(3.73)$ & $1.29(1.97)$ & $1.01(1.20)$ & $<0.001$ \\
\hline Systolic blood pressure, $\mathrm{mmHg}$ & $135(20)$ & $134(20)$ & $134(21)$ & $135(22)$ & 0.43 \\
\hline Diastolic blood pressure, $\mathrm{mmHg}$ & $84(13)$ & $82(12)$ & $82(13)$ & $81(13)$ & $<0.001$ \\
\hline Overweight, \% & 50.2 & 46.4 & 36.9 & 25.0 & $<0.001$ \\
\hline Obesity, \% & 14.1 & 5.9 & 4.1 & 2.7 & $<0.001$ \\
\hline Diabetes, \% & 23.1 & 15.4 & 12.6 & 9.5 & $<0.001$ \\
\hline Hypertension, \% & 56.3 & 51.2 & 49.3 & 51.4 & $<0.05$ \\
\hline Dyslipidemia, $\%$ & 62.6 & 43.3 & 38.7 & 26.0 & $<0.001$ \\
\hline Metabolic syndrome, $\%$ & 37.9 & 24.2 & 20.7 & 11.5 & $<0.001$ \\
\hline
\end{tabular}

The data are summarized as the mean (standard deviation) for continuous variables or as a number with proportion for categorical variables. $P$ for trend was calculated by ANOVA and Chi-square test.

facilitate comparisons across metabolic parameters, association magnitudes are reported in SD units of metabolic parameters per one SD increment in ln-TT [18]. Because TT has a close relationship with MS, which is a confounding factor for other metabolic parameters, we reanalyzed these associations in subjects without MS. The interaction between TT and metabolic syndrome, age category, smoking, and economic and weight status was tested by adding a multiplicative factor in the regression model.

The associations between TT and metabolic diseases (categorical variables) were assessed by logistic regression. The regression models were adjusted for age, smoking, economic status, and BMI (not for obesity indices and MS). Adjusted odds ratios for each one SD increment of ln-TT concentration associated with metabolic diseases in total men and in subgroups were reported.

\section{Results}

3.1. Characteristics of the Study Population by Quartiles of TT. General demographic and laboratory characteristics of the study population are summarized in Table 1 . The quartile ranges were $\leq 12.60,12.61-15.70,15.71-19.91$, and $\geq 19.92 \mathrm{nmol} / \mathrm{L}$. According to the trend analysis, with each decrease in TT by quartile there were significant increases for most metabolic parameters, including BMI, waist and neck circumference, TG, TC, FPG, HbA1c, HOMA-IR, and diastolic blood pressure (all $P$ for trend $<0.001$ ). And as expected, the prevalence of overweight, obesity, dyslipidemia, hypertension, diabetes, and MS also increased through the decreasing TT concentration quartiles (all $P$ for trend $<0.05$ ).

3.2. Association between TT and Metabolic Parameters. Figure 1 summarizes the results of SD units of metabolic parameters per one SD increment in ln-TT, expressed by unstandardized coefficients (B) (95\% confidence interval). Various metabolic measures were associated with TT (10 and 9 measures for total men and men without MS, respectively, at $P<0.05)$. All of the associations were in the same direction for total men and men without MS, though less strong for men without MS. Overall, higher TT was associated with metabolic biomarkers linked with lower cardiometabolic risk. After adjustment for age, smoking, and economic status, BMI (B $-0.32,95 \%$ CI -0.35 to -0.29$)$ had the greatest magnitude of association amongst all metabolic measures. In obesity indices, association magnitudes gradually decreased from BMI, waist circumference, and neck circumference to waist-hip ratio. After adjusting for age, smoking, economic status, and BMI, for the lipid profile, TG ( $\mathrm{B}-0.28,95 \% \mathrm{CI}-0.31$ to -0.25$)$ had the greatest association strength and HDL (B 0.13, 95\%CI 0.10-0.16) had the second greatest. Regarding glycemic indices, FPG 


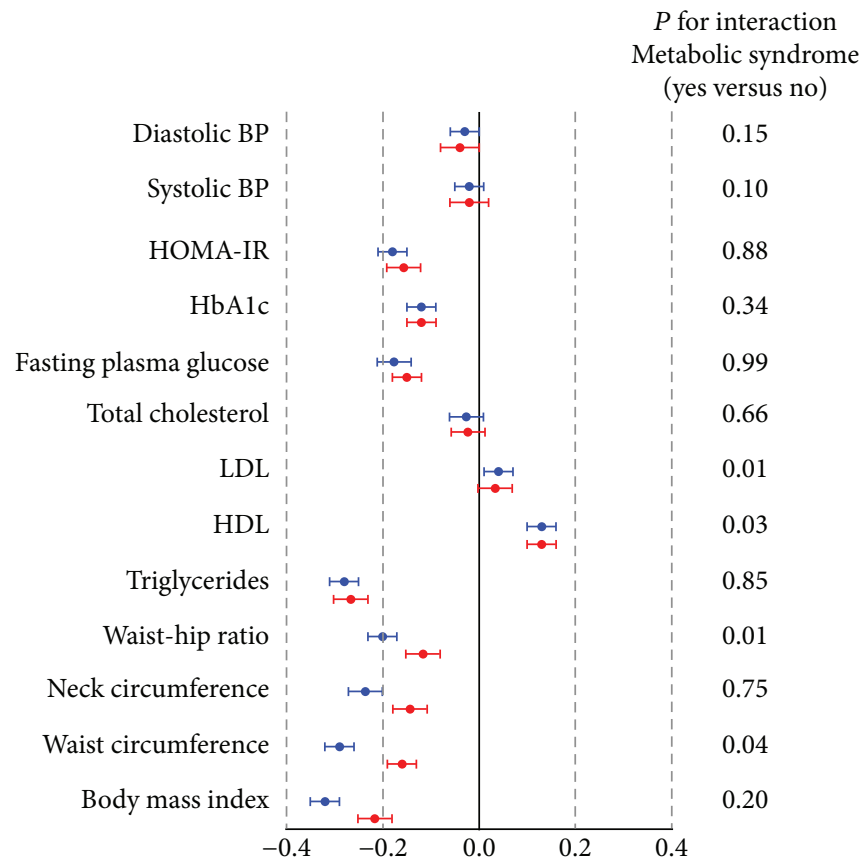

Figure 1: Associations of total testosterone with metabolic measures for total men and men without metabolic syndrome. To facilitate comparisons across metabolic parameters, association magnitudes are reported in SD units of metabolic parameters per one SD increment in ln-total testosterone. Model controls for age, smoking, economic status, and body mass index (not for obesity indices). Blue circles: total subjects; red circles: subject without metabolic syndrome.

(B $-0.18,95 \% \mathrm{CI}-0.21$ to -0.14 ) and HOMA-IR (B -0.18, $95 \% \mathrm{CI}-0.21$ to -0.15$)$ had stronger associations with TT than HbA1c (B -0.12, 95\%CI -0.15 to -0.09$)$. Systolic (B -0.02, 95\%CI -0.05-0.01) and diastolic (B -0.03, 95\%CI -0.06-0.00) blood pressure had a marginal association with TT. The significant interaction effect between MS and TT exists in LDL, HDL, waist-hip ratio, and waist circumference.

3.3. Association between TT and Metabolic Diseases. The associations between TT and metabolic diseases by logistic regression in total subjects and subgroups are listed in Figure 2 and Table 2. After adjusting for age, smoking, economic status, and BMI (not for obesity indices and MS), all the associations of TT with metabolic disorders including MS, obesity, diabetes, hypertension, and dyslipidemia were in the same direction for total men and, if applicable, for men without MS. Obesity defined by BMI (OR 0.40, 95\%CI 0.35-0.45) still had the greatest strength of association, with each one SD increment of TT, whereas hypertension (OR $0.90,95 \%$ CI $0.84-0.98$ ) had the smallest.

According to the stratified analyses, the associations between each one SD increment of TT and the prevalence of MS, overweight, obesity, diabetes, and dyslipidemia were significant in both age strata ( $<60$ and $\geq 60$ years), both current smoking status (yes and no), both economic status (low and high), and both BMI strata $\left(<25\right.$ and $\geq 25 \mathrm{~kg} / \mathrm{m}^{2}$, if applicable). Its association with hypertension was significant in the strata of $<60$ years, nonsmoking, or low economic status. After further interaction analyses, we found that there

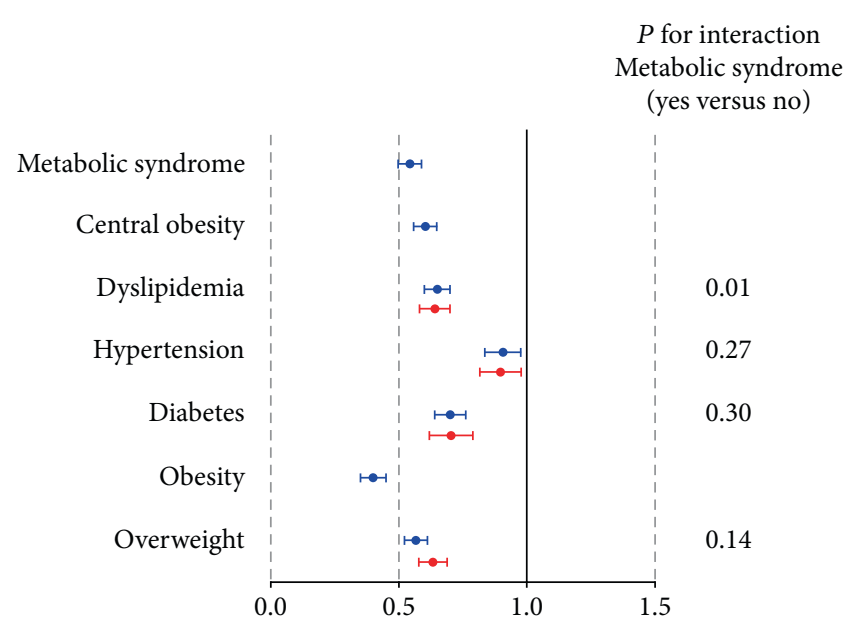

Figure 2: Associations of total testosterone with metabolic diseases for total men and men without metabolic syndrome. Adjusted ORs for each one SD increment of ln-total testosterone associated with metabolic diseases are shown. Logistic regression analysis was used. Model controls for age, smoking, economic status, and body mass index (not for obesity indices and metabolic syndrome). Blue circles: total subjects; red circles: subject without metabolic syndrome.

was no interaction effect between TT and age category, economic, and weight status on metabolic diseases. The main interaction effect on metabolic diseases including obesity indices, dyslipidemia, and metabolic syndrome was between TT and smoking status. 
TABLE 2: Subgroup association of total testosterone with metabolic diseases.

(a)

\begin{tabular}{lcccccc}
\hline & & Age category & & & Smoking status \\
& $<60$ & $\geq 60$ & $P$ for interaction & Nonsmoking & Smoking & $P$ for interaction \\
\hline Overweight & $0.58(0.52,0.65)$ & $0.58(0.51,0.66)$ & 0.37 & $0.61(0.55,0.68)$ & $0.53(0.47,0.60)$ & 0.02 \\
Obesity & $0.39(0.33,0.46)$ & $0.43(0.36,0.53)$ & 0.81 & $0.45(0.38,0.53)$ & $0.36(0.30,0.43)$ & 0.03 \\
Central obesity & $0.61(0.55,0.68)$ & $0.59(0.52,0.67)$ & 0.72 & $0.69(0.62,0.76)$ & $0.54(0.48,0.60)$ & $<0.001$ \\
Diabetes & $0.71(0.62,0.80)$ & $0.70(0.61,0.80)$ & 0.61 & $0.65(0.58,0.74)$ & $0.76(0.67,0.87)$ & 0.28 \\
Hypertension & $0.88(0.80,0.97)$ & $0.94(0.83,1.06)$ & 0.27 & $0.92(0.83,1.02)$ & $0.88(0.79,0.99)$ & 0.88 \\
Dyslipidemia & $0.62(0.56,0.69)$ & $0.71(0.63,0.80)$ & 0.40 & $0.70(0.63,0.77)$ & $0.60(0.54,0.68)$ & 0.02 \\
Metabolic syndrome & $0.56(0.50,0.62)$ & $0.53(0.46,0.60)$ & 0.46 & $0.64(0.57,0.71)$ & $0.46(0.40,0.52)$ & $<0.001$ \\
\hline
\end{tabular}

(b)

\begin{tabular}{|c|c|c|c|c|c|c|}
\hline & \multicolumn{3}{|c|}{ Economic status } & \multicolumn{3}{|c|}{ Weight status } \\
\hline & Low & High & $P$ for interaction & $\mathrm{BMI}<25 \mathrm{~kg} / \mathrm{m}^{2}$ & $\mathrm{BMI} \geq 25 \mathrm{~kg} / \mathrm{m}^{2}$ & $P$ for interaction \\
\hline Overweight & $0.57(0.50,0.64)$ & $0.58(0.52,0.65)$ & 0.24 & & & \\
\hline Obesity & $0.38(0.31,0.46)$ & $0.42(0.35,0.50)$ & 0.27 & & & \\
\hline Central obesity & $0.61(0.55,0.69)$ & $0.59(0.53,0.66)$ & 0.53 & & & \\
\hline Diabetes & $0.70(0.60,0.80)$ & $0.70(0.63,0.79)$ & 0.63 & $0.70(0.62,0.79)$ & $0.65(0.57,0.74)$ & 0.48 \\
\hline Hypertension & $0.91(0.81,1.03)$ & $0.89(0.81,0.98)$ & 0.40 & $0.87(0.79,0.96)$ & $0.82(0.73,0.93)$ & 0.46 \\
\hline Dyslipidemia & $0.68(0.60,0.77)$ & $0.64(0.57,0.71)$ & 0.85 & $0.62(0.55,0.69)$ & $0.61(0.54,0.69)$ & 0.87 \\
\hline Metabolic syndrome & $0.51(0.45,0.58)$ & $0.56(0.50,0.63)$ & 0.27 & & & \\
\hline
\end{tabular}

Adjusted ORs for each one SD increment of ln-total testosterone associated with metabolic diseases. The data are expressed as odds ratio (95\% confidence interval). Logistic regression analysis was used. Model controls for age, smoking, economic status, and body mass index (not for obesity indices and metabolic syndrome).

\section{Discussion}

We found that plasma TT concentrations in Chinese men were significantly associated with various metabolic parameters, as well as diagnosed metabolic diseases. Obesity indices (BMI and waist circumference) and triglycerides had the largest magnitude of association and blood pressure had the smallest. These associations were also present in groups without metabolic syndrome, where obesity is the core component. These results indicated that male low TT could be the result of obesity and diabetes or in some cases contribute to the presence of metabolic disorders.

In this and previous studies, the complex myriad of metabolic perturbations including insulin resistance, dysglycemia, abnormal lipid profile, and adiposity may be intrinsically linked with circulating TT and erectile dysfunction $[5,6,19,20]$. The metabolic profiling here demonstrated that higher TT was related to various metabolic deviations as mentioned above, characterizing a less risk-prone cardiometabolic profile. Overall, a similar type of metabolic perturbations was followed in the metabolic associations with higher TT, as previously reported for a lower degree of adiposity and better lipid and glycemic profile $[5,6,19]$. For example, each 0.41 natural-log nmol/L higher TT level in men was associated with about $1 \mathrm{~kg} / \mathrm{m}^{2}$ lower BMI and $2.3 \mathrm{~cm}$ smaller waist circumference. Furthermore, it is worth mentioning that the metabolite associations with TT remained significant after adjusting for BMI and in individuals without metabolic syndrome where central obesity is the core component. This indicated the associations between TT levels, and numerous metabolic risk perturbations were independent of these measures of adiposity. Though previous studies found that obesity led to lower levels of TT $[21,22]$, this effect was not sufficient enough to explain the strong associations observed between TT and other metabolic parameters independent of obesity. The underlying mechanism remains unclarified.

Obesity indices had bigger association magnitudes than most other metabolic components. Obesity and TT seem to have a bidirectional relationship. Longitudinal studies have found that low TT increases the risk of obesity and metabolic syndrome [5], and conversely, obesity could also lead to low TT $[23,24]$. In a prospective study, obesity-related metabolic and lifestyle factors predispose older men to the development of secondary hypogonadism, which is frequently reversible with weight loss [25]. Moreover, testosterone replacement therapy seems to be an effective approach to achieve sustained weight loss in obese men with low TT [26], though some studies did not achieve it [27], and also conversely, a significant increase in TT levels was induced when weight loss and this increase were greater in those losing more weight [21]. As we mentioned in a previous study [9], obesity and low TT may form a vicious circle. Thus, a combined approach of lifestyle intervention and testosterone treatment might be a further option to break this vicious circle in hypogonadal, obese men $[9,22]$. 
There are preclinical studies exploring the mechanisms how low TT affects glucose and lipid metabolism. Testosterone may improve glucose metabolism by modulating the expression of glucose transporter 4 and insulin receptor and regulating key enzymes involved in glycolysis [28]. Moreover, testosterone could protect murine pancreatic $\beta$ cells against glucotoxicity-induced apoptosis [29]. Regarding lipid metabolism, Santosa and Jensen found that hypogonadal men stored a greater proportion of both dietary fatty acid and free fatty acid in lower body fat than did eugonadal men through upregulating acyl-CoA synthetase activity in femoral adipose tissue [30]. Thus, testosterone could affect body fat distribution. Testosterone also promotes the differentiation of pluripotent stem cells into the myogenic lineage but inhibits their differentiation into adipocytes, which provides the molecular basis for adiposity and insulin resistance [31]. Furthermore, in androgen receptor null male mice, results indicated that androgen receptor is critical in male metabolism by affecting the energy balance and is negative to adiposity [32]. In another simpler rat model, castration induced the expansion of the retroperitoneal fat depot mediated by an increased basal rate of inflammatory cytokine expression and muscle wasting resulting from an inability of leucine to stimulate protein synthesis [33].

It is interesting that we found TT increased with aging in men in our study, which was elaborately discussed in our previous paper [34]. $\mathrm{T}$ is the major androgen in males and 95\% of $\mathrm{T}$ is secreted by Leydig cells, most of which adhere with the sex hormone-binding globulin (SHBG). In fact, $\mathrm{T}$ decline may vary depending on ethnicity, environment, lifestyle, and comorbidities. Some Western studies reported that TT as well as SHBG declined with aging $[35,36]$, while in the Asian some studies found no age-related changes of TT in healthy men [37-39]. Recently, Kelsey et al. [40] analyzed a dataset obtained from 13 studies $(n=10,097$; age range 3-101 years) regardless of ethnicity and found no evidence to support a progressive decline in $\mathrm{T}$ in middle-aged and older men. In our population, age-related increases of SHBG and declining clearance by the kidneys could partially explain the pattern of changes in TT with age in Chinese men [34]. Moreover, we found that the LH levels in Chinese men were positively associated with TT, which suggests that the testicular responsiveness to $\mathrm{LH}$ in Chinese men was not decreased to the same degree as in other populations [34].

Our study had several strengths. First, regarding the significance, this study is the first to analyze the association magnitude between TT and multiple metabolic parameters in general Chinese men, the largest population group in the world. Second, the same trained research group completed the anthropometric measurements and questionnaires in each site, and one laboratory certified by the College of American Pathologists performed all the biomedical measurements, thus strong quality control is guaranteed. Third, our results may be more reflective in a general population as opposed to a clinic-based population.

However, there are also some limitations. First, because this is a cross-sectional nature of the study, we cannot draw a causal relationship between TT and metabolic parameters. Second, the results may not be generalizable to other ethnic groups because of Han Chinese men primarily recruited.

\section{Conclusions}

TT was strongly associated with multiple metabolic parameters reflecting the degree of adiposity, glucose, and lipid metabolism disorders. Obesity indices had closer relationships with TT than most other metabolic measures with blood pressure the least close. These associations remained robust after adjustment for adiposity and in subjects without MS. Study results indicated that low testosterone in male might be screened in individuals with diabetes, dyslipidemia, and especially obesity, regardless of having MS or not.

$\begin{array}{ll}\text { Abbreviations } \\ \text { BMI: } & \text { Body mass index } \\ \text { FPG: } & \text { Fasting plasma glucose } \\ \text { HbA1c: } & \text { Glycated hemoglobin } \\ \text { HDL: } & \text { High-density lipoprotein } \\ \text { HOMA-IR: } & \text { Homeostasis model assessment of insulin } \\ & \text { resistance } \\ \text { LDL: } & \text { Low-density lipoprotein } \\ \text { MS: } & \text { Metabolic syndrome } \\ \text { SD: } & \text { Standard deviation } \\ \text { SPECT-China: } & \text { Survey on Prevalence in East China for Met- } \\ & \text { abolic Diseases and Risk Factors } \\ \text { TC: } & \text { Total cholesterol } \\ \text { TG: } & \text { Triglycerides } \\ \text { TT: } & \text { Total testosterone. }\end{array}$

\section{Data Access}

Please contact Yingli $\mathrm{Lu}$ and Ningjian Wang for data requests.

\section{Ethical Approval}

The study protocol was approved by the Ethics Committee of Shanghai Ninth People's Hospital, Shanghai Jiao Tong University School of Medicine (Approval no. 2013).

\section{Consent}

Informed consent was obtained from all patients included in the study.

\section{Disclosure}

The funders played no role in the design and conduct of the study; collection, management, analysis, and interpretation of data; or preparation, review, and approval of the manuscript.

\section{Conflicts of Interest}

No potential conflicts of interest relevant to this article were reported. 


\section{Authors' Contributions}

Yingli Lu had full access to all the data in the study and takes responsibility for the integrity of the data and the accuracy of the data analysis. Yingli Lu, Ningjian Wang, and Michael Jensen were responsible for the study concept and design. Jing Cheng, Ningjian Wang, Bing Han, Qin Li, Fangzhen Xia, and Hualing Zhai conducted the research. Jing Cheng and Ningjian Wang analyzed the data and drafted the manuscript, which was revised for important intellectual content by all authors. Yingli Lu is the guarantor.

\section{Acknowledgments}

This study was supported by National Natural Science Foundation of China (81570726, 81600609); Shanghai Jiao Tong University School of Medicine (2014); Science and Technology Commission of Shanghai Municipality (16410723200, 16411971200); Shanghai Municipal Commission of Health and Family Planning (20164Y0079); Commission of Health and Family Planning of Pudong District (PW2015D-5); the Fourth Round of Three-Year Public Health Action Plan of Shanghai by the Shanghai Municipal Commission of Health and Family Planning (15GWZK0202); and Clinical Research Plan of SHDC (16CR3076B). The authors thank Xiaojin Wang and Bingshun Wang from the Department of Biostatistics, Shanghai Jiao Tong University School of Medicine, for data processing. The authors thank Weiping $\mathrm{Tu}$, Bin $\mathrm{Li}$, and Ling $\mathrm{Hu}$ for helping organize this investigation. The authors also thank all the team members and participants in SPECT-China study.

\section{References}

[1] J. Lu, L. Wang, M. Li et al., "Metabolic syndrome among adults in China: the 2010 China noncommunicable disease surveillance," The Journal of Clinical Endocrinology \& Metabolism, vol. 102, no. 2, pp. 507-515, 2017.

[2] D. Gu, K. Reynolds, X. Wu et al., "Prevalence of the metabolic syndrome and overweight among adults in China," Lancet, vol. 365, no. 9468, pp. 1398-1405, 2005.

[3] P. F. Corradi, R. B. Corradi, and L. W. Greene, "Physiology of the hypothalamic pituitary gonadal axis in the male," Urologic Clinics of North America, vol. 43, no. 2, pp. 151-162, 2016.

[4] J. J. Cai, J. Wen, W. H. Jiang, J. Lin, Y. Hong, and Y. S. Zhu, "Androgen actions on endothelium functions and cardiovascular diseases," Journal of Geriatric Cardiology, vol. 13, no. 2, pp. 183-196, 2016.

[5] J. S. Brand, M. M. Rovers, B. B. Yeap et al., "Testosterone, sex hormone-binding globulin and the metabolic syndrome in men: an individual participant data meta-analysis of observational studies," PLoS One, vol. 9, no. 7, article e100409, 2014.

[6] R. Blaya, L. D. Thomaz, F. Guilhermano et al., "Total testosterone levels are correlated to metabolic syndrome components," The Aging Male, vol. 19, no. 2, pp. 85-89, 2016.

[7] R. Haring, H. Volzke, S. B. Felix et al., "Prediction of metabolic syndrome by low serum testosterone levels in men: results from the study of health in Pomerania," Diabetes, vol. 58, no. 9, pp. 2027-2031, 2009.
[8] C. Li, E. S. Ford, B. Li, W. H. Giles, and S. Liu, "Association of testosterone and sex hormone-binding globulin with metabolic syndrome and insulin resistance in men," Diabetes Care, vol. 33, no. 7, pp. 1618-1624, 2010.

[9] N. Wang, H. Zhai, B. Han et al., "Visceral fat dysfunction is positively associated with hypogonadism in Chinese men," Scientific Reports, vol. 6, no. 1, article 19844, 2016.

[10] N. Wang, X. Wang, B. Han et al., "Is exposure to famine in childhood and economic development in adulthood associated with diabetes?," The Journal of Clinical Endocrinology \& Metabolism, vol. 100, no. 12, pp. 4514-4523, 2015.

[11] N. Wang, X. Wang, Q. Li et al., "The famine exposure in early life and metabolic syndrome in adulthood," Clinical Nutrition, vol. 36, no. 1, pp. 253-259, 2017.

[12] N. Wang, J. Cheng, B. Han et al., "Exposure to severe famine in the prenatal or postnatal period and the development of diabetes in adulthood: an observational study," Diabetologia, vol. 60, no. 2, pp. 262-269, 2017.

[13] A. Bjornerem, B. Straume, M. Midtby et al., "Endogenous sex hormones in relation to age, sex, lifestyle factors, and chronic diseases in a general population: the Tromso study," The Journal of Clinical Endocrinology \& Metabolism, vol. 89, no. 12, pp. 6039-6047, 2004.

[14] Y. Xu, L. Wang, J. He et al., "Prevalence and control of diabetes in Chinese adults," JAMA, vol. 310, no. 9, pp. 948-959, 2013.

[15] Q. Li, N. Wang, B. Han et al., "Neck circumference as an independent indicator to non-alcoholic fatty liver disease in non-obese men," Nutrition \& Metabolism, vol. 12, no. 1, p. 63, 2015.

[16] J. Lu, Y. Bi, T. Wang et al., "The relationship between insulinsensitive obesity and cardiovascular diseases in a Chinese population: results of the REACTION study," International Journal of Cardiology, vol. 172, no. 2, pp. 388-394, 2014.

[17] K. G. Alberti, P. Zimmet, J. Shaw, and Group IDFETFC, "The metabolic syndrome-a new worldwide definition," Lancet, vol. 366, no. 9491, pp. 1059-1062, 2005.

[18] Q. Wang, A. J. Kangas, P. Soininen et al., "Sex hormonebinding globulin associations with circulating lipids and metabolites and the risk for type 2 diabetes: observational and causal effect estimates," International Journal of Epidemiology, vol. 44, no. 2, pp. 623-637, 2015.

[19] B. B. Yeap, S. A. Chubb, Z. Hyde et al., "Lower serum testosterone is independently associated with insulin resistance in non-diabetic older men: the Health In Men Study," European Journal of Endocrinology, vol. 161, no. 4, pp. 591598, 2009.

[20] G. Corona, S. Cipriani, G. Rastrelli, A. Sforza, E. Mannucci, and M. Maggi, "High triglycerides predicts arteriogenic erectile dysfunction and major adverse cardiovascular events in subjects with sexual dysfunction," The Journal of Sexual Medicine, vol. 13, no. 9, pp. 1347-1358, 2016.

[21] G. Corona, G. Rastrelli, M. Monami et al., "Body weight loss reverts obesity-associated hypogonadotropic hypogonadism: a systematic review and meta-analysis," European Journal of Endocrinology, vol. 168, no. 6, pp. 829-843, 2013.

[22] G. Corona, L. Vignozzi, A. Sforza, E. Mannucci, and M. Maggi, "Obesity and late-onset hypogonadism," Molecular and Cellular Endocrinology, vol. 418, Part 2, pp. 120-133, 2015.

[23] C. A. Derby, S. Zilber, D. Brambilla, K. H. Morales, and J. B. McKinlay, "Body mass index, waist circumference and waist to hip ratio and change in sex steroid hormones: the 
Massachusetts Male Ageing Study," Clinical Endocrinology, vol. 65, no. 1, pp. 125-131, 2006.

[24] E. M. Camacho, I. T. Huhtaniemi, T. W. O'Neill et al., “Ageassociated changes in hypothalamic-pituitary-testicular function in middle-aged and older men are modified by weight change and lifestyle factors: longitudinal results from the European Male Ageing Study," European Journal of Endocrinology, vol. 168, no. 3, pp. 445-455, 2013.

[25] G. Rastrelli, E. L. Carter, T. Ahern et al., "Development of and recovery from secondary hypogonadism in aging men: prospective results from the EMAS," The Journal of Clinical Endocrinology \& Metabolism, vol. 100, no. 8, pp. 3172-3182, 2015.

[26] F. Saad, A. Yassin, G. Doros, and A. Haider, "Effects of longterm treatment with testosterone on weight and waist size in 411 hypogonadal men with obesity classes I-III: observational data from two registry studies," International Journal of Obesity, vol. 40, no. 1, pp. 162-170, 2016.

[27] K. S. Nair, R. A. Rizza, P. O'Brien et al., "DHEA in elderly women and DHEA or testosterone in elderly men," New England Journal of Medicine, vol. 355, no. 16, pp. 1647-1659, 2006.

[28] P. M. Rao, D. M. Kelly, and T. H. Jones, “Testosterone and insulin resistance in the metabolic syndrome and T2DM in men," Nature Reviews Endocrinology, vol. 9, no. 8, pp. 479493, 2013.

[29] W. Hanchang, N. Semprasert, T. Limjindaporn, P. T. Yenchitsomanus, and S. Kooptiwut, "Testosterone protects against glucotoxicity-induced apoptosis of pancreatic $\beta$-cells (INS-1) and male mouse pancreatic islets," Endocrinology, vol. 154, no. 11, pp. 4058-4067, 2013.

[30] S. Santosa and M. D. Jensen, "Effects of male hypogonadism on regional adipose tissue fatty acid storage and lipogenic proteins," PLoS One, vol. 7, no. 2, article e31473, 2012.

[31] R. Singh, J. N. Artaza, W. E. Taylor, N. F. Gonzalez-Cadavid, and S. Bhasin, "Androgens stimulate myogenic differentiation and inhibit adipogenesis in $\mathrm{C} 3 \mathrm{H} 10 \mathrm{~T} 1 / 2$ pluripotent cells through an androgen receptor-mediated pathway," Endocrinology, vol. 144, no. 11, pp. 5081-5088, 2003.

[32] T. Yanase, W. Fan, K. Kyoya et al., “Androgens and metabolic syndrome: lessons from androgen receptor knock out (ARKO) mice," The Journal of Steroid Biochemistry and Molecular Biology, vol. 109, no. 3-5, pp. 254-257, 2008.

[33] Q. Jiao, A. M. Pruznak, D. Huber, T. C. Vary, and C. H. Lang, "Castration differentially alters basal and leucine-stimulated tissue protein synthesis in skeletal muscle and adipose tissue," American Journal of Physiology Endocrinology and Metabolism, vol. 297, no. 5, pp. E1222-E1232, 2009.

[34] F. Xia, N. Wang, B. Han et al., "Hypothalamic-pituitarygonadal axis in aging men and women: increasing total testosterone in aging men," Neuroendocrinology, vol. 104, no. 3, pp. 291-301, 2017.

[35] J. E. Morley, F. E. Kaiser, H. M. Perry 3rd et al., "Longitudinal changes in testosterone, luteinizing hormone, and folliclestimulating hormone in healthy older men," Metabolism, vol. 46, no. 4, pp. 410-413, 1997.

[36] S. M. Harman, E. J. Metter, J. D. Tobin, J. Pearson, and M. R. Blackman, "Longitudinal effects of aging on serum total and free testosterone levels in healthy men. Baltimore longitudinal study of aging," The Journal of Clinical Endocrinology \& Metabolism, vol. 86, no. 2, pp. 724-731, 2001.
[37] M. Taya, E. Koh, K. Izumi et al., "Comparison of testosterone fractions between Framingham Heart Study participants and Japanese participants," International Journal of Urology, vol. 21, no. 7, pp. 689-695, 2014.

[38] J. Zhao and C. M. Schooling, "Does falling testosterone with age among men underlie the increase in ischaemic heart disease," Journal of Epidemiology and Community Health, vol. 69, no. 4, pp. 393-396, 2015.

[39] Z. Liu, J. Liu, X. Shi, L. Wang, Y. Yang, and M. Tao, "Dynamic alteration of serum testosterone with aging: a cross-sectional study from Shanghai, China," Reproductive Biology and Endocrinology, vol. 13, no. 1, p. 111, 2015.

[40] T. W. Kelsey, L. Q. Li, R. T. Mitchell, A. Whelan, R. A. Anderson, and W. H. Wallace, "A validated age related normative model for male total testosterone shows increasing variance but no decline after age 40 years," PLoS One, vol. 9, no. 10, article e109346, 2014. 


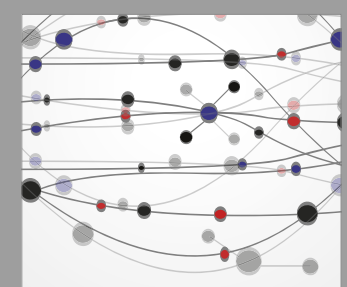

The Scientific World Journal
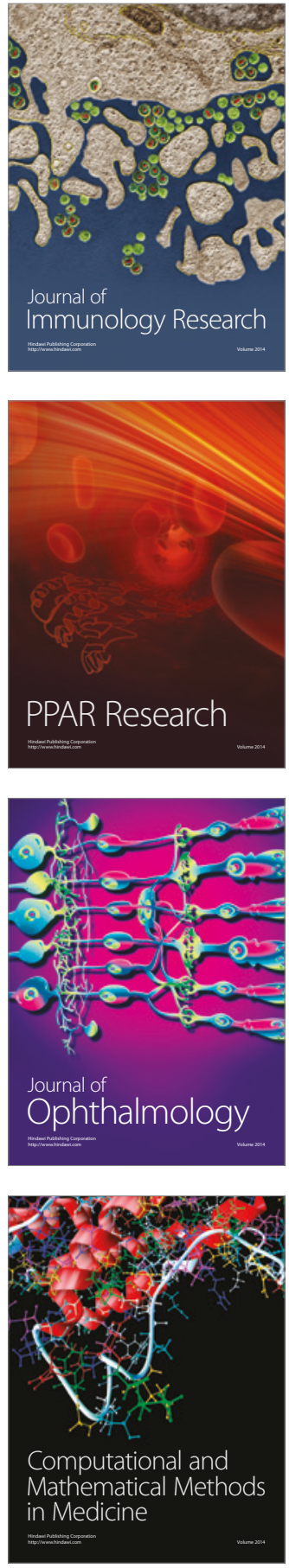

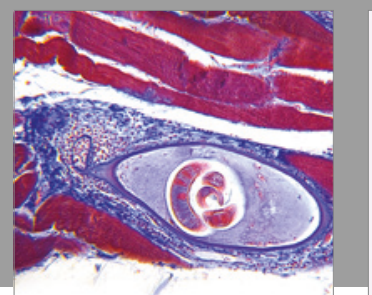

Gastroenterology Research and Practice
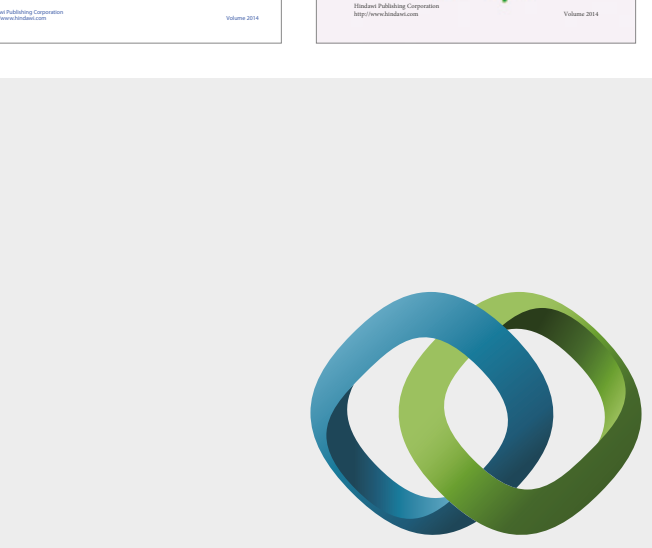

\section{Hindawi}

Submit your manuscripts at

https://www.hindawi.com
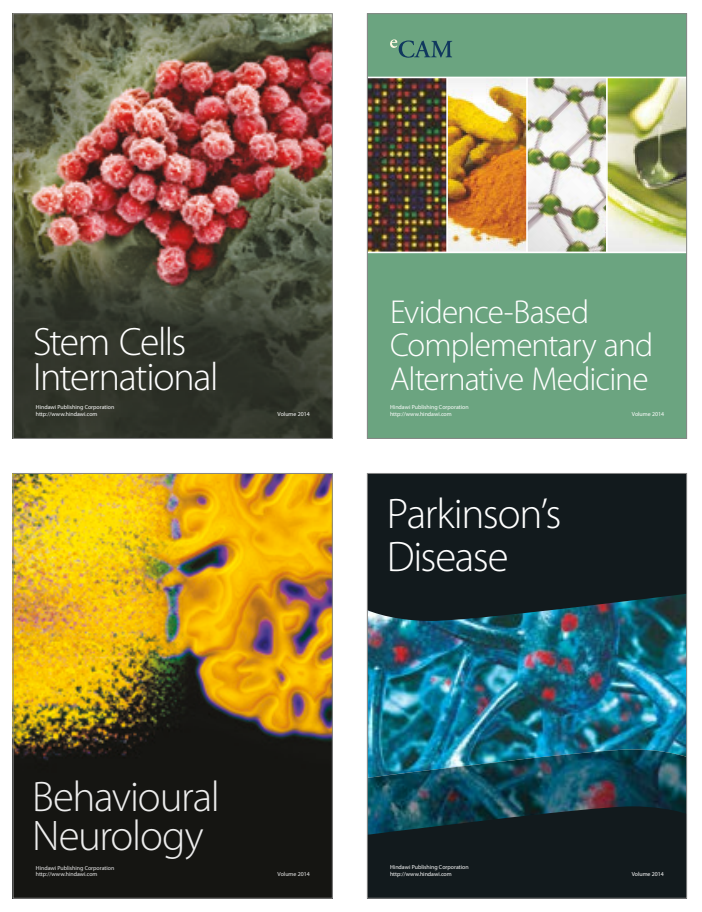
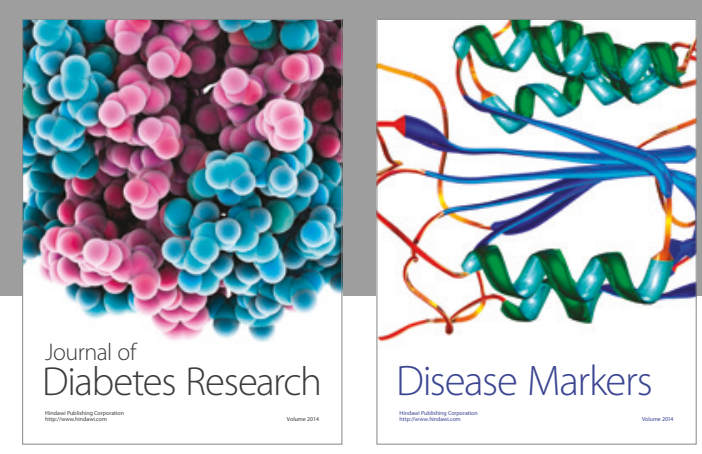

Disease Markers
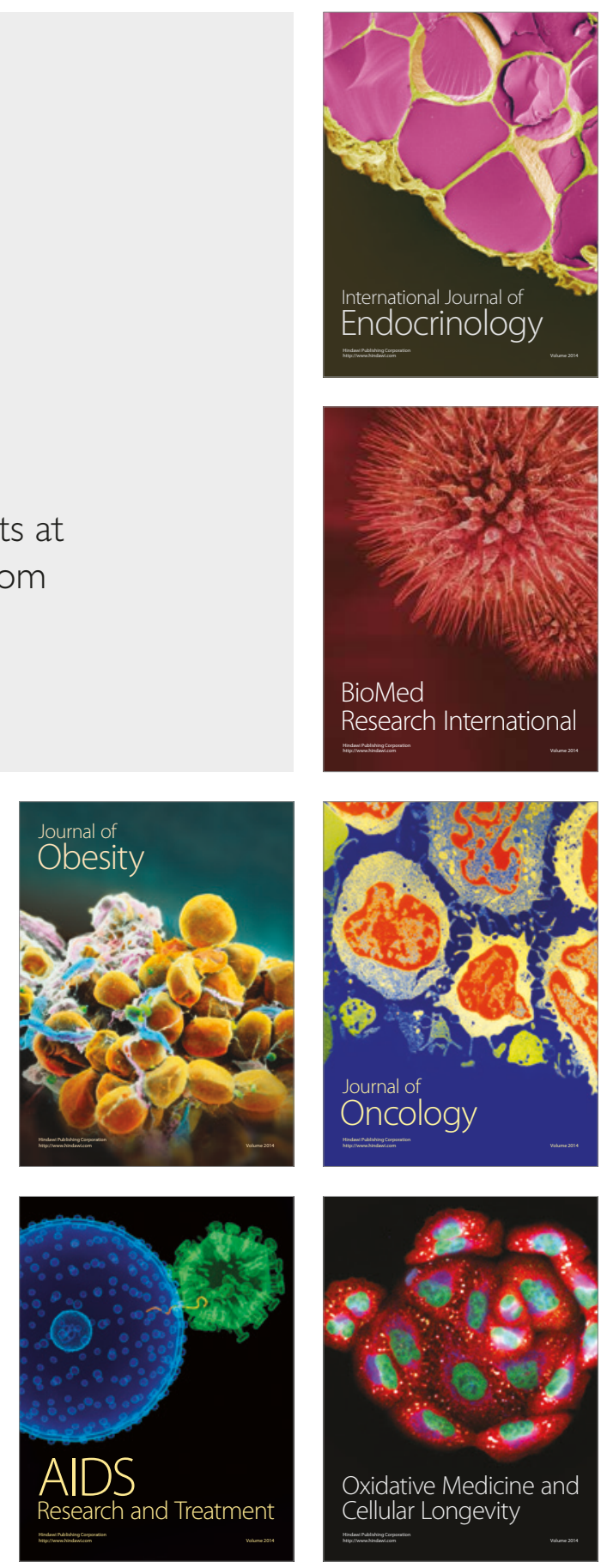